\author{
Shaohua Chen ${ }^{1}$ \\ LNM, \\ Institute of Mechanics, \\ Chinese Academy of Sciences, \\ Beijing 100190, China \\ e-mail: chenshaohua72@hotmail.com \\ Yin Yao \\ LNM, \\ Institute of Mechanics, \\ Chinese Academy of Sciences, \\ Beijing 100190, China \\ e-mail: yaoyin111@LNM.imech.ac.cn
}

\section{Elastic Theory of Nanomaterials Based on Surface-Energy Density}

Recent investigations into surface-energy density of nanomaterials lead to a ripe chance to propose, within the framework of continuum mechanics, a new theory for nanomaterials based on surface-energy density. In contrast to the previous theories, the linearly elastic constitutive relationship that is usually adopted to describe the surface layer of nanomaterials is not invoked and the surface elastic constants are no longer needed in the new theory. Instead, a surface-induced traction to characterize the surface effect in nanomaterials is derived, which depends only on the Eulerian surface-energy density. By considering sample-size effects, residual surface strain, and external loading, an explicit expression for the Lagrangian surface-energy density is achieved and the relationship between the Eulerian surface-energy density and the Lagrangian surface-energy density yields a conclusion that only two material constants-the bulk surface-energy density and the surface-relaxation parameter-are needed in the new elastic theory. The new theory is further used to characterize the elastic properties of several fcc metallic nanofilms under biaxial tension, and the theoretical results agree very well with existing numerical results. Due to the nonlinear surface effect, nanomaterials may exhibit a nonlinearly elastic property though the inside of nanomaterials or the corresponding bulk one is linearly elastic. Moreover, it is found that externally applied loading should be responsible for the softening of the elastic modulus of a nanofilm. In contrast to the surface elastic constants required by existing theories, the bulk surface-energy density and the surface-relaxation parameter are much easy to obtain, which makes the new theory more convenient for practical applications. [DOI: 10.1115/1.4028780]

Keywords: nanomaterials, surface effect, surface-energy density, surface-relaxation parameter

\section{Introduction}

Although many physical mechanisms can induce sizedependent phenomena in nanomaterials, one of the most important mechanisms is the effect of free surfaces [1-3]. Compared to large-scale bulk materials, nanomaterials exhibit a large surfaceto-volume ratio. Atoms at or near a free surface of a nanomaterial may experience a different local environment compared with atoms in the interior of the nanomaterial, inducing charge redistributions and changes in the length of atomic bonds [4]. Consequently, the equilibrium position and energy of each atom near the surface can differ significantly from those in the interior. Such effects have been identified as a main mechanism leading to the size effects associated with the elastic moduli, resonant frequency, and thermal conductivity of nanoscale materials [5-8].

Many experiments have explored the surface effect in nanomaterials, including characterizing surface atomic structures in various nanomaterials by using electron diffraction and scanningprobe microscopy [9-12] and by measuring the size-dependent mechanical properties of nano-sized structural elements [13-17]. The experimental findings provide a convincing demonstration that surface effects play an important role in the mechanical properties of nanomaterials.

The theoretical analysis of surface properties began about a century ago. The approach then was to characterize the physical changes of atoms at or near a solid surface by two continuum quantities: the surface free energy and the surface stress $[18,19]$.

\footnotetext{
${ }^{1}$ Corresponding author.

Contributed by the Applied Mechanics of ASME for publication in the JournaL of Applied Mechanics. Manuscript received July 9, 2014; final manuscript received October 6, 2014; accepted manuscript posted October 10, 2014; published online October 20, 2014. Editor: Yonggang Huang.
}

Later, Gurtin and Murdoch established a rigorous mathematical framework to model the surface elasticity (G-M theory) [20,21], in which a linearized constitutive law was assumed to describe a surface layer with vanishing thickness. A set of surface elastic constants, analogous to the bulk elastic constants, were introduced to characterize the surface properties, leading to a surface stress-strain relationship similar in form to the generalized Hooke's law. The surface (or interface) stress-strain constitutive expressions were further investigated by Nix and Gao [22] and Haiss [23] from the atomic point of view. A detailed discussion of surface elastic theory can be found in Refs. [3] and [24].

Based on surface elastic theory, a large number of theoretical models have been successfully created to analyze surface effects in nanomaterials. Steigmann and Ogden [25] and Chhapadia et al. [26] modified G-M theory by introducing a surface flexural stiffness into the constitutive model, with the aim being to characterize the dependence of surface energy on curvature due to bending or wrinkling of nanowires (NWs). Wang and Feng [27,28] and He and Lilley [29,30] adopted Young-Laplace (Y-L) equation to study static and dynamic bending of NWs, where the effect of surface stress induced by the curvature was taken into account. Song et al. [31] further studied the same problem with a modified Y-L model that considered the in-plane surface stress tangential to the longitudinal surfaces of a NW. The mechanical behavior of a nano-thin film was investigated by $\mathrm{Lim}$ and $\mathrm{He}$ [32], Lu et al. [33], and Huang [34], incorporating the surface elasticity into classical plate theory. Lachut and Sader [35] explored the effect of surface stress on the stiffness of a cantilever nanoplate. Dingreville et al. $[36,37]$ formulated the surface elastic constants in tensorial form and discussed the surface effect with respect to the elastic properties of various nanostructures under general loading conditions. Huang and Wang [38] proposed a hyperelastic surface model to study how the surface effect induces radial shrinkage in 
a nanoparticle. G-M theory has also been successfully extended to the interface effect in nanocomposites [39-47]. A comprehensive review on the theory of surface elasticity and its applications on nanostructured elements have been given by Wang et al. recently [48].

In addition to the theoretical models that have been developed to describe the surface effect of nanomaterials, numerical investigations have also progressed greatly. These mainly focus on molecular dynamics (MD) simulations to describe the inevitable surface effect on the atomic scale. In two representative studies, Miller and Shenoy [5] and Shenoy [49] calculated the surface elastic constants of typically metallic materials. Other work in this area was done by Streitz et al. [50,51], Diao et al. [52], Cao and Chen [53], Mi et al. [54], On et al. [55], Pahlevani and Shodja [56], Mohammadi and Sharma [57], Olsson and Park [58], and others. Gao et al. [59], Park and Klein [60], and Liu et al. [61] also developed the finite-element method to study surface effects. From the numerical calculations, one can see that the required surface elastic constants are a little bit difficult to determine $[5,26,49,54,58]$.

It is well known that the energy of each atom in the surface region deviates significantly from those in the interior of a nanomaterial, and this discrepancy has been identified as the main cause of size effects of nanomaterials. Furthermore, in recent years, the surface-energy density of nanomaterials was deeply studied and clarified [22,62-67], which greatly facilitates investigating surface effects in nanomaterials from the point of view of energy.

In this paper, we propose a new elastic theory for nanomaterials that is based on the surface-energy density. Only the bulk surfaceenergy density and relaxation parameter are needed to characterize the surface properties of a nanosolid. Theoretical predictions of the mechanical behavior of typical metallic nanofilms agree well with existing numerical results. Furthermore, the stiffening and softening mechanisms of a nanofilm subjected to a uniform tension are also disclosed.

\section{Nanomaterial Surface-Energy Density}

2.1 Lagrangian Surface-Energy Density $\phi_{0}$. Consider a nanosolid with the initial (or reference) configuration shown in Fig. 1, which consists of a domain $V_{0}$ in a three-dimensional Euclidean space bounded by the surface $S_{0}$. The body force $\mathbf{f}$ and the surface traction $\mathbf{p}$ induce a displacement $\mathbf{u}$ and strain $\boldsymbol{\varepsilon}$, which lead to the present configuration that occupies the domain $V$ bounded by surface $S$. Transformation from the reference configuration $V_{0}$ to the present configuration $V$ passes through an intermediate configuration $V_{\mathrm{r}}$, which corresponds to the nanosolid experiencing a surface residual strain induced only by surface relaxation. Assume that the material has an idealized crystal structure and that the reference configuration consists of a planar array of atoms with a surface area $A_{0}$, as shown in Fig. 2. A Lagrangian coordinate system is embedded in the deformed surface and attached to the atoms [22], with the principal axes 1 and 2 parallel to the two basic vectors of the surface unit cell. The quantities $a_{01}$, $a_{02}$, and $N_{0}$ represent the lattice lengths in the two principal directions and the number of atoms, respectively. $\beta$ denotes the angle between the two basic vectors and is $90 \mathrm{deg}$ in most cases. Transforming $V_{0}$ to $V_{\mathrm{r}}$ leads to an intermediate area $A_{\mathrm{r}}$, with the atomic spacings becoming $a_{\mathrm{r} 1}$ and $a_{\mathrm{r} 2}$. Under external loading, the atomic spacings are further modified and become $a_{1}$ and $a_{2}$ in the present surface element, which has surface area $A$. Without considering any chemical reaction or phase transformation, the number $N_{0}$ of atoms and the angle $\beta$ remain unchanged during the entire process.

According to Nix and Gao [22], the excess free energy per surface atom is $g_{x s}^{0}$. Considering both the effect of surface relaxation and that of external loading, the free energy per surface atom can be written as

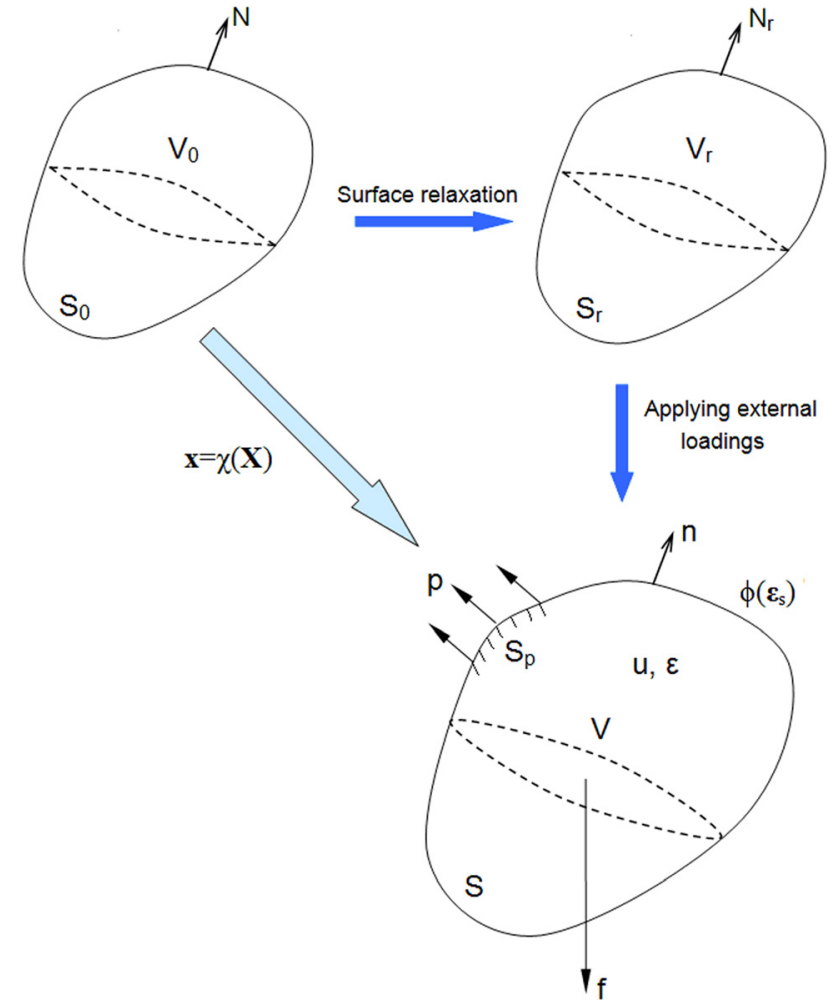

Fig. 1 Reference, intermediate, and present configurations of a three-dimensional nanosolid with the corresponding volumes and surface areas $V_{0}$ and $S_{0}, V_{\mathrm{r}}$ and $S_{\mathrm{r}}, V$ and $S$, respectively. $P$ is a surface traction that acts on the surface area $S_{P}$, and $f$ is a body force. The quantities $u$ and $\varepsilon$ are the displacement and strain fields, respectively.

$$
g_{x s}^{1}=g_{x s}^{0}+\frac{1}{2} \sum_{i=1}^{2} k_{i}\left(a_{i}-a_{0 i}\right)^{2}
$$

where $k_{i}(i=1,2)$ are spring constants in the two principal directions $[22,64]$.

The surface-energy density in $V_{0}$ (i.e., the Lagrangian surfaceenergy density) is defined as

$$
\phi_{0}=\frac{N_{0} g_{x s}^{1}}{A_{0}}=\frac{N_{0} g_{x s}^{1}}{N_{0} a_{01} a_{02} \sin \beta}=\phi_{0}^{*}+\frac{1}{2} \sum_{i=1}^{2} k_{i} \frac{\left(a_{i}-a_{0 i}\right)^{2}}{a_{01} a_{02} \sin \beta}
$$

where $\phi_{0}^{*}=g_{x s}^{0} /\left(a_{01} a_{02} \sin \beta\right)$.

The surface-energy density in $V$ (i.e., the Eulerian surfaceenergy density) is

$$
\phi=\frac{N_{0} g_{x S}^{1}}{A}=\phi_{0} \frac{A_{0}}{A}
$$

The ratio of $A$ to $A_{0}$ is

$$
\frac{A}{A_{0}}=\left(1+\varepsilon_{t 1}\right)\left(1+\varepsilon_{t 2}\right)=J_{\mathrm{s}}
$$

where $\varepsilon_{t 1}=\left(a_{1}-a_{01}\right) / a_{01}$ and $\varepsilon_{t 2}=\left(a_{2}-a_{02}\right) / a_{02}$ represent the surface strains in the bond directions and $J_{s}$ is a Jacobian determinant characterizing the deformation between $V_{0}$ and $V$.

Thus, we have

$$
\phi=\phi_{0} / J_{s}
$$



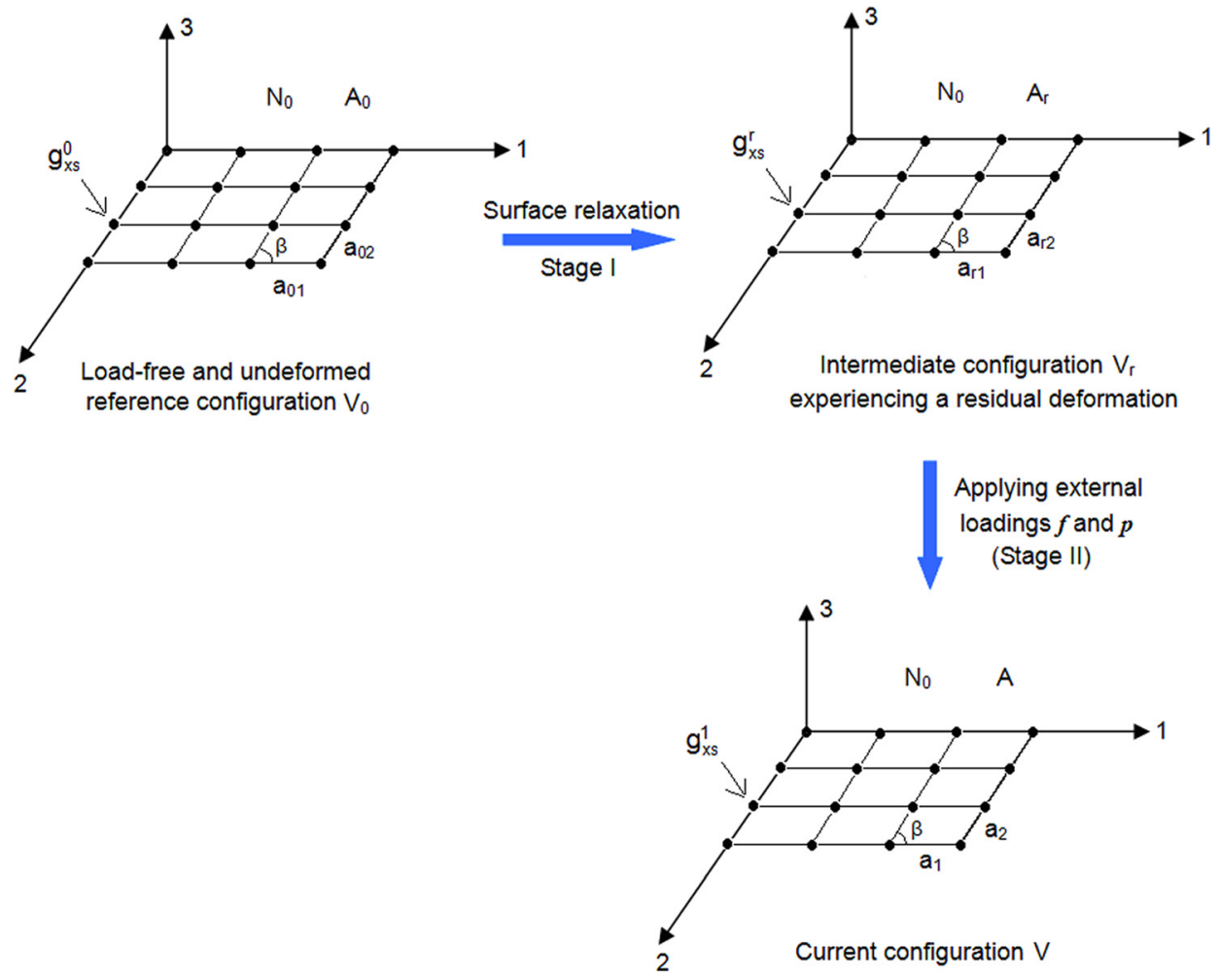

Fig. 2 Schematic of a surface element in the reference, intermediate, and present configurations

(i) Considering the effect of surface relaxation alone, we have

$$
a_{\mathrm{r} 1}=\lambda_{1} a_{01}, \quad a_{\mathrm{r} 2}=\lambda_{2} a_{02}
$$

where $\lambda_{1}$ and $\lambda_{2}$ are two surface relaxation parameters in the two principal directions. $\lambda_{i}>1$ denotes a surface stretched due to dilation and $\lambda_{i}<1$ denotes a surface compressed due to shrinkage. The surface residual strains are defined as

$$
\varepsilon_{\mathrm{ri}}=\frac{a_{\mathrm{r} i}-a_{0 i}}{a_{0 i}}=\lambda_{i}-1, \quad(i=1,2)
$$

(ii) Under external loading, the surface strains induced only by external loading are defined as

$$
\varepsilon_{s i}=\frac{a_{i}-a_{\mathrm{r} i}}{a_{\mathrm{r} i}}, \quad(i=1,2)
$$

Here, surface deformation of nanosolids in the present theory consults Nix and Gao [22] and Ouyang et al. [64], where the concepts of Lagrangian, intermediate, and Eulerian configurations are similarly used as those in Huang and Wang [38]. Surface strains in local coordinate system are related to the deformation of lattice length in two directions [22]. It is reasonable for an elastic surface with infinitesimal deformation from the atomic point of view, though different from the continuum mechanics definition in terms of displacement gradient [68]. Surface strain in the local coordinate system can be transferred to the surface strain tensor in a global coordinate system in G-M theory [20,21].

Next, the second term on the right side of Eq. (2) in directions 1 and 2 can be further written as

$\frac{1}{2} k_{1} \frac{\left(a_{1}-a_{01}\right)^{2}}{a_{01} a_{02} \sin \beta}=\frac{k_{1}}{2 \sin \beta} \frac{a_{01}}{a_{02}}\left[\lambda_{1}^{2} \varepsilon_{s 1}^{2}+2 \lambda_{1}\left(\lambda_{1}-1\right) \varepsilon_{s 1}+\left(\lambda_{1}-1\right)^{2}\right]$ and

$\frac{1}{2} k_{2} \frac{\left(a_{2}-a_{02}\right)^{2}}{a_{01} a_{02} \sin \beta}=\frac{k_{2}}{2 \sin \beta} \frac{a_{02}}{a_{01}}\left[\lambda_{2}^{2} \varepsilon_{s 2}^{2}+2 \lambda_{2}\left(\lambda_{2}-1\right) \varepsilon_{s 2}+\left(\lambda_{2}-1\right)^{2}\right]$

In a perfect-crystal lattice structure, interactions between atoms can be characterized by a spring constant $k_{0}$ [69], which is the product of the bulk Young's modulus and the bulk lattice constant (i.e., $k_{0}=E_{0} a_{0}$ ). Based on this, Sun et al. [62] and Ouyang et al. [64] proposed the following similar expression for the spring constants in the two principal directions of a surface unit cell:

$$
k_{i}=E_{\mathrm{A} i} a_{0 i}, \quad(i=1,2)
$$

where $E_{\mathrm{A} i}$ is the Young's modulus in each principal direction of the surface unit cell. According to the model involving bond order, bond length, and bond strength [62,63], $E_{\mathrm{A} i}$ is written as

$$
E_{\mathrm{A} i}=E_{0}\left[\left(\frac{a_{i}}{a_{0 i}}\right)^{-m}-3\left(\frac{a_{i}}{a_{0 i}}\right)+3\right], \quad(i=1,2)
$$

where $m$ is a parameter describing the dependence of bond length on the binding energy: $m=-4$ for alloys or compounds and $m=1$ for pure metals.

Thus, spring constants in the surface region can be written as

$$
k_{i}=E_{0} a_{0 i}\left[\left(\lambda_{i}+\lambda_{i} \varepsilon_{s i}\right)^{-m}-3 \lambda_{i}\left(1+\varepsilon_{s i}\right)+3\right], \quad(i=1,2)
$$

The Lagrangian surface energy density becomes

$$
\begin{aligned}
\phi_{0}= & \phi_{0}^{*}+\frac{E_{0}}{2 \sin \beta} \sum_{i=1}^{2} a_{0 i} \eta_{i}\left[\left(\lambda_{i}+\lambda_{i} \varepsilon_{s i}\right)^{-m}-3 \lambda_{i}\left(1+\varepsilon_{s i}\right)+3\right] \\
& \times\left[\lambda_{i}^{2} \varepsilon_{s i}^{2}+\left(\lambda_{i}-1\right)^{2}+2 \lambda_{i}\left(\lambda_{i}-1\right) \varepsilon_{s i}\right]
\end{aligned}
$$

where $\eta_{1}=a_{01} / a_{02}$ and $\eta_{2}=a_{02} / a_{01}$. 
2.2 Size-Dependent Characteristic of $\phi_{0}^{*}$. The second term on the right of Eq. (2), as a structural part, is related to the surface strain energy, whereas the first term $\phi_{0}^{*}$ on the right of Eq. (2), as a chemical part, originates from the surface dangling-bond energy [64-66]. Thus, we have [64,70,71]

$$
\phi_{0}^{*}=\frac{Z_{\mathrm{b}}-Z_{s}}{Z_{\mathrm{b}}} \frac{U_{\mathrm{coh}}(D)}{a_{01} a_{02} \sin \beta}
$$

where $Z_{\mathrm{b}}$ and $Z_{s}$ are the coordination numbers per bulk and surface atom, $Z_{\mathrm{b}}-Z_{s}$ denotes the number of broken bonds at the surface (dangling bonds), $a_{01} a_{02} \sin \beta$ represents the area of the surface unit cell, and $U_{\mathrm{coh}}(D)$ is the cohesive energy of an atom in the nanocrystal, which depends on a characteristic scale $D$ of the nanostructure (e.g., thickness, diameter, etc.) [72].

The bulk surface-energy density $\phi_{0 \mathrm{~b}}$ can be expressed as [70]

$$
\phi_{0 \mathrm{~b}}=\frac{Z_{\mathrm{b}}-Z_{s}}{Z_{\mathrm{b}}} \frac{U_{\mathrm{coh}}^{\mathrm{b}}}{a_{01} a_{02} \sin \beta}
$$

where $U_{\text {coh }}^{\mathrm{b}}$ is the cohesive energy per bulk atom.

According to the classical thermodynamic models [73], the size dependence of $U_{\text {coh }}(D)$ is

$$
\frac{U_{\mathrm{coh}}(D)}{U_{\mathrm{coh}}^{\mathrm{b}}}=\left[1-\frac{1}{w_{1} D / D_{0}-1}\right] \exp \left(-\frac{3 S_{\mathrm{b}}}{2 R} \frac{1}{w_{1} D / D_{0}-1}\right)
$$

where $S_{\mathrm{b}}$ and $R$ represent the bulk melting entropy and the ideal gas constant, respectively, and $D_{0}$ is the critical size $\left(D_{0}=3 d_{0}\right.$ and $2 d_{0}$ for a nanoparticle and a nanothin film, respectively, where $d_{0}$ is the atomic diameter). The quantity $w_{1}$ is a positive parameter with different values for different nano-elements (particle, wire, film, etc.).

Combining Eqs. (15)-(17) yields the size-dependent chemical surface-energy density

$$
\phi_{0}^{*}=\phi_{0 \mathrm{~b}}\left[1-\frac{1}{w_{1} D / D_{0}-1}\right] \exp \left(-\frac{3 S_{\mathrm{b}}}{2 R} \frac{1}{w_{1} D / D_{0}-1}\right)
$$

which can be approximated as $[67,71]$

$$
\phi_{0}^{*}=\phi_{0 \mathrm{~b}}\left(1-\frac{D_{0}}{w_{2} D}\right), \quad w_{2}=\frac{3 R w_{1}}{2 S_{\mathrm{b}}}
$$

Equations (19) are analogous to the well-known Tolman equation [74]. For most fcc metals, $3 R / 2 S_{\mathrm{b}} \sim 1.2[75]$ and $w_{2}$ depends on $w_{1}$, which is given in the literatures $[64,65,67]$.

The Lagrangian surface-energy density in Eq. (14) can be further written as

$$
\begin{aligned}
\phi_{0}= & \phi_{0 \mathrm{~b}}\left(1-\frac{D_{0}}{w_{2} D}\right)+\frac{E_{0}}{2 \sin \beta} \sum_{i=1}^{2} a_{0 i} \eta_{i} \\
& \times\left\{\left[3+\left(\lambda_{i}+\lambda_{i} \varepsilon_{\mathrm{si}}\right)^{-m}-3\left(\lambda_{i}+\lambda_{i} \varepsilon_{\mathrm{s} i}\right)\right]\right. \\
& \left.\times\left[\lambda_{i}^{2} \varepsilon_{\mathrm{s} i}^{2}+\left(\lambda_{i}-1\right)^{2}+2 \lambda_{i}\left(\lambda_{i}-1\right) \varepsilon_{\mathrm{s} i}\right]\right\}
\end{aligned}
$$

Here, it is interesting that the Lagrangian surface-energy density of a nanomaterial involves two material parameters: the bulk surface energy density $\phi_{0 \mathrm{~b}}$ and the surface relaxation parameters $\lambda_{i}$, both of which have clear physical meanings and are very easy to obtain by experiment or atomistic simulation.

\section{New Elastic Theory Based on Surface-Energy Density}

3.1 Equilibrium Equations and Boundary Conditions. As shown in Fig. 1, the relationship between the reference and present configurations can be characterized by a mapping function $\mathbf{x}=\chi(\mathbf{X})$, where $\mathbf{x}$ and $\mathbf{X}$ represent position vectors of a material point in the present and reference configurations, respectively.

In the present configuration, the strain fields in the bulk and on the surface can be expressed as

$$
\begin{aligned}
\boldsymbol{\varepsilon}(\mathbf{x}) & =(\mathbf{u} \otimes \nabla+\nabla \otimes \mathbf{u}) / 2, \quad \mathbf{x} \in V-S \\
\boldsymbol{\varepsilon}_{s} & =\boldsymbol{\varepsilon}\left(\mathbf{x}_{s}\right), \quad \mathbf{x}_{s}=\left.\mathbf{x}\right|_{\text {on } S}=\theta_{\alpha} \mathbf{e}_{\alpha}+r \mathbf{n} \quad(\alpha=1,2 ; r=\mathrm{const})
\end{aligned}
$$

where $\nabla$ is a spatial gradient operator with respect to $\mathbf{x}, \mathbf{x}_{s}$ represents the position vector of a material point on $S, \mathbf{e}_{\alpha}$, and $\theta_{\alpha}$ denote unit vectors and coordinates in two orthogonal principal directions of $S$, respectively, $\mathbf{n}=\mathbf{n}\left(\theta_{1}, \theta_{2}\right)$ is the unit normal vector perpendicular to $S$, and the normal coordinate $r$ remains constant on $S$ [68]. The potential energy function $\Pi$ of the solid consists of three parts: the bulk elastic strain energy $U$, the surface free energy $\Phi$, and the external work $W$, which can be written as $[24,32,76]$

$$
\begin{aligned}
\Pi(\mathbf{u}) & =U+\Phi-W \\
& =\int_{V-S} \rho \psi(\boldsymbol{\varepsilon}) d V+\int_{S} \phi\left(\boldsymbol{\varepsilon}_{S}\right) d S-\int_{V-S} \mathbf{f} \cdot \mathbf{u} d V-\int_{S_{p}} \mathbf{p} \cdot \mathbf{u} d S
\end{aligned}
$$

where $\psi$ is the elastic strain energy density and $\rho$ is the mass density. The quantity $\phi$ depends not only on the position vector $\mathbf{x}_{s}$ but also on the surface strain $\varepsilon_{s} ; d V$ and $d S$ are the infinitesimal volume and surface area, respectively, and $S_{p}\left(S_{p} \subseteq S\right)$ represents the region of the boundary where an external traction $\mathbf{p}$ acts.

Variational analysis of Eq. (22) yields [38]

$$
\left\{\begin{array}{l}
\boldsymbol{\sigma} \cdot \nabla+\mathbf{f}=0 \quad(\text { in } V-S) \\
\mathbf{n} \cdot \boldsymbol{\sigma} \cdot \mathbf{n}=\mathbf{p} \cdot \mathbf{n}+\boldsymbol{\sigma}_{s}: \mathbf{b} \quad(\text { on } S) \\
(\mathbf{I}-\mathbf{n} \otimes \mathbf{n}) \cdot \boldsymbol{\sigma} \cdot \mathbf{n}=(\mathbf{I}-\mathbf{n} \otimes \mathbf{n}) \cdot \mathbf{p}+\boldsymbol{\sigma}_{s} \cdot \nabla_{s} \quad(\text { on } S) \\
\boldsymbol{\sigma}_{s} \cdot \mathbf{n}_{l}=0 \quad(\text { on } \partial S)
\end{array}\right.
$$

where $\boldsymbol{\sigma}$ and $\boldsymbol{\sigma}_{s}$ are the bulk and the surface Cauchy stress tensors, respectively, $\mathbf{b}$ is the curvature tensor of $S, \partial S$ is a closed smooth curve enclosing $S$ with $\mathbf{I}$ and $\mathbf{n}_{l}$ being its unit tangential and normal vectors, $\mathbf{I}$ is a unit tensor, and $\nabla_{s}$ is a surface gradient operator with respect to $\mathbf{x}_{S}$ on $S$. The derivation used the inner products $\mathbf{n} \cdot \mathbf{n}_{l}=0, \mathbf{n} \cdot \mathbf{e}_{\alpha}=0$, and $\mathbf{n} \cdot \delta \mathbf{u}_{\mathrm{t}}=0$.

The second and third equations in Eq. (23) denote the stress boundary conditions in the normal and tangential directions of surface $S$, respectively, and can be rewritten as

$$
\begin{aligned}
\boldsymbol{\sigma} \cdot \mathbf{n} & =\mathbf{p}-\boldsymbol{\gamma} \quad(\text { on } S) \\
\boldsymbol{\gamma} & =\gamma_{\mathrm{n}} \mathbf{n}+\gamma_{\mathrm{t}}, \quad \gamma_{\mathrm{n}}=-\boldsymbol{\sigma}_{s}: \mathbf{b}, \quad \gamma_{\mathrm{t}}=-\boldsymbol{\sigma}_{s} \cdot \nabla_{s}
\end{aligned}
$$

Here, it is interesting that, in contrast to the result with classical continuum mechanics, an additional traction vector $\gamma$ is introduced into the stress boundary conditions to account for the surface effect of nanomaterials. Furthermore, the relationship between $\gamma_{\mathrm{n}}$ and $\gamma_{\mathrm{t}}$, and the surface stress $\boldsymbol{\sigma}_{s}$ abides by the generalized Young-Laplace equations [38]. Here, we call $\gamma$ the "surfaceinduced traction," which is a force disturbance at a boundary due to the surface effect.

Variation of the surface-free energy can be further expressed as

$$
\begin{aligned}
\delta \Phi & =\int_{S} \boldsymbol{\sigma}_{S}:(\delta \mathbf{u} \otimes \nabla) d S \\
& =-\int_{S}\left[\delta \mathbf{u}_{\mathrm{t}} \cdot\left(\boldsymbol{\sigma}_{s} \cdot \nabla_{s}\right)+\delta u_{\mathrm{n}}\left(\boldsymbol{\sigma}_{s}: \mathbf{b}\right)\right] d S=\int_{S} \boldsymbol{\gamma} \cdot \delta \mathbf{u} d S
\end{aligned}
$$




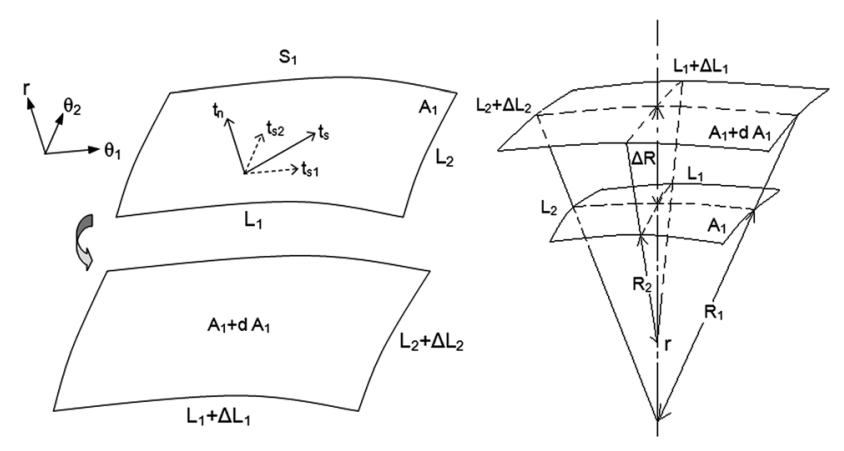

Fig. 3 Schematic of an infinitesimal area element after deformation. The boundary lengths $L_{1}$ and $L_{2}$ change to become $L_{1}+\Delta L_{1}$ and $L_{2}+\Delta L_{2} . \Delta R$ is the normal displacement of the curved surface. The initial surface area $A_{1}$ changes to become $A_{1}+d A_{1}$.

where $\delta \mathbf{u}=\delta \mathbf{u}\left(\mathbf{x}_{s}\right)$ represents the variational displacement of a material point on surface $S$. Equation (25) indicates that the surface-energy variation due to surface deformation equals the work done by the surface-induced traction [77].

3.2 Surface-Induced Traction $\gamma$. As shown in Fig. 3, an infinitesimal area element $S_{1}$ is taken out of the present curved surface $S$. The position of a material point on $S_{1}$ is described by a curvilinear coordinate system $\left(\theta_{1}, \theta_{2}, r\right)$. Let $L_{1}$ and $L_{2}$ represent the boundary arc lengths; the area of $S_{1}$ is expressed as $A_{1}=H L_{1} L_{2}$, where $H$ is a coefficient. With Eq. (24), the resultant traction $\mathbf{t}$ on $S_{1}$ can be obtained as [78,79]

$$
\mathbf{t}=[\boldsymbol{\sigma}] \cdot \mathbf{n}=\left(\boldsymbol{\sigma}_{+}-\boldsymbol{\sigma}_{-}\right) \cdot \mathbf{n}=\mathbf{p}-\boldsymbol{\sigma} \cdot \mathbf{n}=\boldsymbol{\gamma}
$$

where $\boldsymbol{\sigma}_{+}$and $\boldsymbol{\sigma}_{-}$represent stresses above and below the surface $S_{1}$, respectively. Equation (26) gives a vanishing net force on $S_{1}$ if the surface effect is not considered. The surface-induced traction $\gamma$ leads to a stress discontinuity across the surface.

Consider an admissible virtual displacement field $\delta \mathbf{s}=\left(\Delta L_{1}\right.$, $\left.\Delta L_{2}, \Delta R\right)$ on $S_{1}$, where $\Delta L_{1}$ and $\Delta L_{2}$ are in-plane components of $\delta \mathbf{s}$ in the two principal directions of $S_{1}$ and $\Delta R$ denotes the normal displacement of the curved surface (see Fig. 3). The virtual work done by the external force on the element can be expressed as

$$
\begin{aligned}
\Delta W & =\mathbf{t} A_{1} \cdot \delta \mathbf{s} \\
& =\left(t_{s 1} \Delta L_{1}+t_{s 2} \Delta L_{2}+t_{\mathrm{n}} \Delta R\right) A_{1} \\
& =\left(\gamma_{1} \Delta L_{1}+\gamma_{2} \Delta L_{2}+\gamma_{\mathrm{n}} \Delta R\right) A_{1}
\end{aligned}
$$

where $\gamma_{1}$ and $\gamma_{2}$ denote the in-plane components of the surfaceinduced traction vector $\gamma$ and $\gamma_{\mathrm{n}}$ is the normal component of the surface-induced traction.

The surface deformation $\delta$ s leads to changes in both the surface-energy density $\phi$ and the area of element $A_{1}$, which are denoted by $d \phi$ and $d A_{1}$, respectively. Neglecting the infinitesimal quantity, $\Delta \Phi$ can be written as

$$
\Delta \Phi=(\phi+d \phi)\left(A_{1}+d A_{1}\right)-\phi A_{1}=d\left(\phi A_{1}\right)
$$

The change of $A_{1}$ is

$$
d A_{1}=H\left(L_{1} \Delta L_{2}+L_{2} \Delta L_{1}\right)
$$

Using the energy-balance relationship, $\Delta W=\Delta \Phi$ yields

$$
\gamma_{1} \Delta L_{1}+\gamma_{2} \Delta L_{2}+\gamma_{\mathrm{n}} \Delta R=\phi\left(\frac{\Delta L_{1}}{L_{1}}+\frac{\Delta L_{2}}{L_{2}}\right)+d \phi
$$

According to Ref. [80], the in-plane stretching or shrinkage of a curved surface will lead to changes of the principal radii of curvature $R_{1}$ and $R_{2}$. The geometric relationship in Fig. 3 gives [80]

$$
\Delta L_{1} / L_{1}=\Delta R / R_{1}, \quad \Delta L_{2} / L_{2}=\Delta R / R_{2}
$$

Considering the dependence of $\phi$ on the position $\left(\theta_{1}, \theta_{2}\right)$ yields

$$
d \phi=\frac{\partial \phi}{\partial \theta_{1}} d \theta_{1}+\frac{\partial \phi}{\partial \theta_{2}} d \theta_{2}
$$

where $d \theta_{1}$ and $d \theta_{2}$ in the two orthogonal principal directions of the surface are equivalent to the infinitesimal increments $\Delta L_{1}$ and $\Delta L_{2}$, respectively. Substituting Eqs. (31) and (32) into Eq. (30) leads to

$$
\gamma_{1}=\frac{\partial \phi}{\partial \theta_{1}}, \quad \gamma_{2}=\frac{\partial \phi}{\partial \theta_{2}}, \quad \gamma_{\mathrm{n}}=\phi\left(\frac{1}{R_{1}}+\frac{1}{R_{2}}\right)
$$

For a curved surface, $\mathbf{n} \cdot \nabla_{s}=\left(1 / R_{1}+1 / R_{2}\right)$. Then, we have

$$
\gamma_{\mathrm{t}}=\nabla_{s} \phi, \gamma_{\mathrm{n}} \mathbf{n}=\phi\left(\frac{1}{R_{1}}+\frac{1}{R_{2}}\right) \mathbf{n}=\phi\left(\mathbf{n} \cdot \nabla_{s}\right) \mathbf{n}
$$

The surface-induced traction can thus be expressed as a function of surface-energy density instead of surface stress as in $\mathrm{G}-\mathrm{M}$ theory, which is the main difference between the present theory and the well-known continuum ones $[3,20,36,38]$. The tangential component $\gamma_{t}$ equals the gradient of the surface-energy density, which is a general relationship between the potential energy and the associated conservative forces. While the formula of $\gamma_{n}$ is the well-known Young-Laplace equation [80], which is responsible for the normal surface dilatation or shrinkage with a nonzero curvature.

Considering the relationship between the Eulerian and Lagrangian surface-energy densities leads to

$$
\gamma_{\mathrm{t}}=\nabla_{s} \phi=\frac{\nabla_{s} \phi_{0}}{J_{s}}-\frac{\phi_{0}\left(\nabla_{s} J_{s}\right)}{J_{s}^{2}}, \quad \gamma_{\mathrm{n}} \mathbf{n}=\frac{\phi_{0}\left(\mathbf{n} \cdot \nabla_{s}\right) \mathbf{n}}{J_{s}}
$$

The equilibrium equations and boundary conditions can be further written as

$$
\left\{\begin{array}{l}
\boldsymbol{\sigma} \cdot \nabla+\mathbf{f}=0 \quad(\text { in } V-S) \\
\mathbf{n} \cdot \boldsymbol{\sigma} \cdot \mathbf{n}=\mathbf{p} \cdot \mathbf{n}-\frac{\phi_{0}\left(\mathbf{n} \cdot \nabla_{s}\right)}{J_{s}} \quad(\text { on } S) \\
(\mathbf{I}-\mathbf{n} \otimes \mathbf{n}) \cdot \boldsymbol{\sigma} \cdot \mathbf{n}=(\mathbf{I}-\mathbf{n} \otimes \mathbf{n}) \cdot \mathbf{p}+\frac{\phi_{0}\left(\nabla_{s} J_{s}\right)}{J_{s}^{2}}-\frac{\nabla_{s} \phi_{0}}{J_{s}}
\end{array}\right.
$$

In contrast to $\mathrm{G}-\mathrm{M}$ theory $[20,21]$, the Lagrangian surface-energy density $\phi_{0}$ in the reference configuration serves as a unique quantity that characterizes the surface effect of nanomaterials. It depends on both the bulk surface-energy density and the relaxation parameter.

\section{Surface Effect of Nanofilm Under Biaxial Tension}

Consider a square nanofilm of thickness $h$, as shown in Fig. 4. A rectangular coordinate system $\left\{x_{1}, x_{2}, x_{3}\right\}$ is introduced, where the $x_{1} O x_{2}$ plane coincides with the midplane of the film. A uniform biaxial tension $\sigma$ is applied on both lateral surfaces $x_{1}=0$, $x_{1}=b$ and $x_{2}=0, x_{2}=b$, which induces a tensile strain field $\varepsilon$. The upper and lower boundary surfaces $x_{3}= \pm h / 2$ are traction free. 


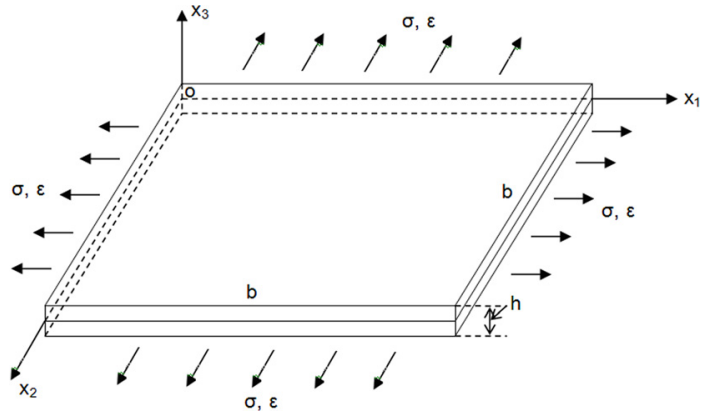

Fig. 4 Square-shaped nanofilm subjected to biaxial tension $\sigma$. The edge length is $b$ and the thickness is $h$. As a result, the strain due to external biaxial tension is $\varepsilon$.

Within the framework of classical elastic theory, we have

$$
\sigma=E_{b} \varepsilon, \quad E_{b}=\frac{E_{0}}{1-\nu_{0}}
$$

where $E_{b}, E_{0}$, and $\nu_{0}$ are the biaxial modulus, Young's modulus, and Poisson's ratio of a bulk material, respectively.

Consider the surface effect of the nanofilm. Let $\sigma_{1}, \varepsilon_{1}, u_{1}$ and $\sigma_{2}, \varepsilon_{2}, u_{2}$ denote the tensile stresses, strains, and displacements in the $x_{1}$ and $x_{2}$ directions, respectively. We have

$$
\sigma_{1}=\sigma_{2}=\sigma, \quad \varepsilon_{1}=\varepsilon_{2}=\varepsilon, \quad \varepsilon_{1}=\frac{\partial u_{1}}{\partial x_{1}}, \quad \varepsilon_{2}=\frac{\partial u_{2}}{\partial x_{2}}
$$

The variation of the bulk strain energy is

$$
\delta U=b h\left[\int_{0}^{b} E_{b} \frac{\partial u_{1}}{\partial x_{1}} \frac{\partial\left(\delta u_{1}\right)}{\partial x_{1}} d x_{1}+\int_{0}^{b} E_{b} \frac{\partial u_{2}}{\partial x_{2}} \frac{\partial\left(\delta u_{2}\right)}{\partial x_{2}} d x_{2}\right]
$$

Since the thickness $h$ of the nanofilm is much less than the width $b$, only the surface effect on the upper and lower surfaces is considered $[32,36]$.

The variation of the surface energy can be written as

$$
\delta \Phi=\int_{S_{f}} \boldsymbol{\gamma} \cdot \delta \mathbf{u} d S=2 b\left(\int_{0}^{b} \gamma_{1} \delta u_{1} d x_{1}+\int_{0}^{b} \gamma_{2} \delta u_{2} d x_{2}\right)
$$

where $S_{f}$ represents the upper and lower surfaces, $\gamma_{\mathrm{n}}$ vanishes due to a zero surface curvature, and

$$
\gamma_{i}=\frac{1}{J_{s}} \frac{\partial \phi_{0}}{\partial x_{i}}-\frac{\phi_{0}}{J_{s}^{2}} \frac{\partial J_{s}}{\partial x_{i}} \quad(i=1,2)
$$

For simplicity, the upper and lower surfaces are assumed to be symmetric and have equal atom spacings in both bond directions (e.g., the (001) or (111) surfaces). As a result, $a_{01}=a_{02}=a_{0 s}$, $\lambda_{1}=\lambda_{2}=\lambda$, and $\varepsilon_{s i}=\varepsilon(i=1,2)[51,52,64,65]$. In addition, we have $D=h, m=1$ and the critical size $D_{0}=2 d_{0}$, where $d_{0}$ is the atomic diameter of the metallic material. For the present fcc metallic-nanofilm case, $w_{2} \approx 4[81,82]$.

For this case, the Lagrangian surface-energy density can be written as

$$
\begin{aligned}
\phi_{0}= & \phi_{0 \mathrm{~b}}\left(1-\frac{d_{0}}{2 h}\right)+\frac{E_{0} a_{0 s}}{\sin \beta}\left[3+\frac{1}{\lambda+\lambda \varepsilon}-3(\lambda+\lambda \varepsilon)\right] \\
& \times\left[\lambda^{2} \varepsilon^{2}+2 \lambda(\lambda-1) \varepsilon+(\lambda-1)^{2}\right]
\end{aligned}
$$

Ignoring the high-order terms, $\phi_{0}$ can be further simplified to

$$
\begin{aligned}
\phi_{0}= & \phi_{0 \mathrm{~b}}\left(1-\frac{d_{0}}{2 h}\right)+\frac{E_{0} a_{0 s}}{\sin \beta}(\lambda-1)^{2}+\frac{E_{0} a_{0 s}}{\sin \beta} \\
& \times\left\{\left[1-10(\lambda-1)-17(\lambda-1)^{2}\right] \varepsilon^{2}\right. \\
& \left.+\left[2(\lambda-1)-10(\lambda-1)^{2}\right] \varepsilon\right\}
\end{aligned}
$$

and

$$
\begin{aligned}
\frac{\partial \phi_{0}}{\partial x_{i}}= & \frac{2 E_{0} a_{0 s} \varepsilon}{\sin \beta}\left[1-10(\lambda-1)-17(\lambda-1)^{2}\right] \frac{\partial \varepsilon}{\partial x_{i}} \quad i=1,2 \\
& +\frac{E_{0} a_{0 s}}{\sin \beta}\left[2(\lambda-1)-10(\lambda-1)^{2}\right] \frac{\partial \varepsilon}{\partial x_{i}}
\end{aligned}
$$

Within the scope of a small deformation, the Jacobian determinant $J_{s}$ is

$J_{s}=J_{s 1} J_{s 2}, \quad J_{s 1}=\left(1+\varepsilon_{r}\right)^{2}, \quad J_{s 2}=\left(1+\varepsilon_{s 1}\right)\left(1+\varepsilon_{s 2}\right)=(1+\varepsilon)^{2}$

Ignoring the high-order strain terms $(n \geq 2)$ yields

$$
J_{s} \approx 1+2\left(\varepsilon_{r}+\varepsilon\right)=1+2(\varepsilon+\lambda-1)
$$

whose partial derivatives with respect to $x_{1}$ and $x_{2}$ are

$$
\frac{\partial J_{s}}{\partial x_{i}}=2 \frac{\partial \varepsilon}{\partial x_{i}} \quad(i=1,2)
$$

Applying the Taylor series approximation leads to

$$
\begin{aligned}
& \frac{1}{J_{s}} \approx 1-\left(\frac{1}{J_{s}^{2}}\right)_{J_{s}=1}\left(J_{s}-1\right)=1-2\left(\varepsilon_{\mathrm{r}}+\varepsilon\right) \\
& \frac{1}{J_{s}^{2}} \approx 1-\left(\frac{2}{J_{s}^{3}}\right)_{J_{s}=1}\left(J_{s}-1\right)=1-4\left(\varepsilon_{\mathrm{r}}+\varepsilon\right)
\end{aligned}
$$

Next, the surface-induced tractions can be obtained as

$$
\begin{aligned}
\gamma_{i} & =\left(f_{1}+f_{2} \varepsilon\right) \frac{\partial^{2} u_{i}}{\partial x_{i}^{2}}, \quad(i=1,2) \\
f_{1} & =\frac{E_{0} a_{0 s}}{\sin \beta}\left[2(\lambda-1)-10(\lambda-1)^{2}\right](3-2 \lambda)-2 \phi_{0 \mathrm{~d}}^{*}(5-4 \lambda) \\
f_{2} & =8 \phi_{0 \mathrm{~d}}^{*}+\frac{2 E_{0} a_{0 s}}{\sin \beta}\left[1-16(\lambda-1)+31(\lambda-1)^{2}\right] \\
\phi_{0 \mathrm{~d}}^{*} & =\phi_{0 \mathrm{~b}}\left(1-\frac{d_{0}}{2 h}\right)+\frac{E_{0} a_{0 s}}{\sin \beta}(\lambda-1)^{2}
\end{aligned}
$$

Combining Eqs. (39), (40), and (49) yields

$$
\begin{aligned}
\delta \Pi= & \delta U+\delta \Phi-\delta W \\
= & b h\left[\int_{0}^{b} E_{b} \frac{\partial u_{1}}{\partial x_{1}} \frac{\partial\left(\delta u_{1}\right)}{\partial x_{1}} d x_{1}+\int_{0}^{b} E_{b} \frac{\partial u_{2}}{\partial x_{2}} \frac{\partial\left(\delta u_{2}\right)}{\partial x_{2}} d x_{2}\right] \\
& +2 b\left[\int_{0}^{b}\left(f_{1}+f_{2} \varepsilon\right) \frac{\partial^{2} u_{1}}{\partial x_{1}^{2}} \delta u_{1} d x_{1}+\int_{0}^{b}\left(f_{1}+f_{2} \varepsilon\right) \frac{\partial^{2} u_{2}}{\partial x_{2}^{2}} \delta u_{2} d x_{2}\right] \\
& -b h\left(\left.\sigma \delta u_{1}\right|_{x_{1}=0}+\left.\sigma \delta u_{1}\right|_{x_{1}=b}+\left.\sigma \delta u_{2}\right|_{x_{2}=0}+\left.\sigma \delta u_{2}\right|_{x_{2}=b}\right)
\end{aligned}
$$


which is further reduced to

$$
\begin{aligned}
\delta \Pi= & -\int_{0}^{b}\left[E_{b} b h-2 b\left(f_{1}+f_{2} \varepsilon\right)\right] \frac{\partial^{2} u_{1}}{\partial x_{1}^{2}} \delta u_{1} d x_{1} \\
& -\int_{0}^{b}\left[E_{b} b h-2 b\left(f_{1}+f_{2} \varepsilon\right)\right] \frac{\partial^{2} u_{2}}{\partial x_{2}^{2}} \delta u_{2} d x_{2} \\
& +b h\left[\left.\left(E_{b} \frac{\partial u_{1}}{\partial x_{1}}-\sigma\right) \delta u_{1}\right|_{x_{1}=0}+\left.\left(E_{b} \frac{\partial u_{2}}{\partial x_{2}}-\sigma\right) \delta u_{2}\right|_{x_{2}=0}\right. \\
& \left.+\left.\left(E_{b} \frac{\partial u_{1}}{\partial x_{1}}-\sigma\right) \delta u_{1}\right|_{x_{1}=b}+\left.\delta u_{2}\right|_{x_{2}=b}\right]=0 \\
& +\left(E_{b} \frac{\partial u_{2}}{\partial x_{2}}-\sigma\right)
\end{aligned}
$$

The equilibrium equation for a nanofilm under a biaxial tension can be obtained as

$$
E_{b}\left[1-\frac{2\left(f_{1}+f_{2} \varepsilon\right)}{E_{b} h}\right] \frac{\partial^{2} u_{i}}{\partial x_{i}^{2}}=0
$$

Comparing Eq. (52) to the classical equilibrium equation for a thin film under a biaxial tension leads to

$$
E_{\mathrm{eff}} \frac{\partial^{2} u_{i}}{\partial x_{i}^{2}}=0 \quad(i=1,2)
$$

where the effective biaxial modulus $E_{\text {eff }}$ is

$$
E_{\text {eff }}=E_{b}\left(1-\frac{2 f_{1}}{E_{b} h}-\frac{2 f_{2} \varepsilon}{E_{b} h}\right)
$$

From Eqs. (49) and (54), it is interesting to find that the effective biaxial modulus of a nanofilm depends not only on the thickness but also on the surface relaxation parameter and the externally applied load. It means that the surface layer of nanomaterials may be nonlinearly elastic though the inside of nanomaterials is elastic as a bulk elastic material. As a result, the whole property may exhibit a nonlinearly elastic one for nanomaterials. Generally, in the case without an external loading, surface relaxation (or residual stress) will influence the lattice length of atoms near the surface, which results in significant size effect in nanomaterials due to the large surface-to-volume ratio. With an external loading, the lattice length will be further influenced, which will show obvious effects on the whole mechanical properties of nanomaterials. In contrast, for a bulk elastic material, the effect of surface is very weak and can be neglected. The nonlinear elasticity of nanomaterials is due to the nonlinearly elastic boundary condition on surfaces. Actually, such a conclusion is consistent well with the results of existing MD simulations [51,52,83], in which the numerically obtained moduli of nanofilms and NWs exhibit obvious dependence on externally applied strains. When the surface effect is weakened with an increasing characteristic size (e.g., the film thickness $h$ ), the effect of strain on the elastic modulus of materials tends to vanish, and a linearly elastic property is exhibited in a bulk state. Therefore, the surface effect at nanoscale can induce not only a size effect but also a nonlinearly elastic property of nanomaterials. It can be inferred that the nonlinearly elastic property of nanomaterials would become strong under a relatively large loading or for a small characteristic size. A theory of hyperelasticity should be adopted if surface effect of nanomaterials is considered from the surface elastic theory point of view [38].
Table 1 Bulk surface-energy density $\phi_{0 \mathrm{~b}}$ and $c_{1}[49,51]$

\begin{tabular}{ccccc}
\hline \hline & $\phi_{0 \mathrm{~b}(001)}(\mathrm{N} / \mathrm{m})$ & $\phi_{0 \mathrm{~b}(111)}(\mathrm{N} / \mathrm{m})$ & $c_{1(001)}(\mathrm{nm})$ & $c_{1(111)}(\mathrm{nm})$ \\
\hline $\mathrm{Cu}$ & 1.33 & 1.24 & 0.012 & 0.009 \\
$\mathrm{Ag}$ & 0.87 & 0.8 & 0.016 & 0.013 \\
$\mathrm{Au}$ & 0.8 & 0.7 & 0.025 & 0.019 \\
\hline \hline
\end{tabular}

Table 2 Atomic diameter, lattice constant, Young's modulus, Poisson's ratio, and biaxial modulus of bulk fcc metals $[64,84]$

\begin{tabular}{lllccc}
\hline \hline & $d_{0}(\mathrm{~nm})$ & $a_{0}(\mathrm{~nm})$ & $E_{0}(\mathrm{GPa})$ & $\nu_{0}$ & $E_{\mathrm{b}}(\mathrm{GPa})$ \\
\hline $\mathrm{Cu}$ & 0.255 & 0.3615 & 110 & 0.34 & 166.7 \\
$\mathrm{Ag}$ & 0.2889 & 0.418 & 78 & 0.37 & 123.8 \\
$\mathrm{Au}$ & 0.2884 & 0.42 & 79 & 0.42 & 136.2 \\
\hline \hline
\end{tabular}

\section{Results and Discussion}

We now use the new theory to predict the biaxial moduli of copper, silver, and gold nanofilms with (001) or (111) surface orientation. The atomic spacing of the surface unit cell is $a_{0 s}=\sqrt{2} a_{0} / 2$, where $a_{0}$ is the bulk lattice constant of a material. On the (001) surface, $\beta=90 \mathrm{deg}$, whereas $\beta=60 \mathrm{deg}$ for the (111) surface. It is proven that the surface residual strain $\varepsilon_{\mathrm{r}}$ is inversely proportional to the film's thickness (i.e., $\varepsilon_{\mathrm{r}}=-c_{1} / h$ $\left.\left(c_{1}>0\right)\right)$ [50,52], which leads to $\lambda=1-c_{1} / h$. The bulk surfaceenergy density and $c_{1}$ for the (001) and (111) surfaces are listed in Table $1[49,51]$. The other material parameters involved in our model are given in Table $2[64,84]$.

5.1 Theoretical Versus MD Results Without External Loading. In previous atomistic simulations, the biaxial modulus of a nanofilm was always obtained without external loading [51,52]. Accordingly, $E_{\text {eff }}$ in Eq. (54) reduces to

$$
E_{\mathrm{eff}}=E_{b}\left(1-\frac{2 f_{1}}{E_{b} h}\right)
$$

Based on Eq. (55), Figs. 5 and 6 present the size-dependent biaxial moduli of copper, silver, and gold nanofilms for (001) and (111) surface orientations, respectively. In these figures, MD results are also shown for comparisons [51,52]. Both the theoretical predictions and the MD results show a monotonic decrease of the nanofilms' biaxial modulus with an increasing film thickness. The modulus approaches the bulk modulus when the film thickness $h$ exceeds about $3 \mathrm{~nm}$. The results show that, for a load-free nanofilm with a contractive surface relaxation $\left(\lambda<1\right.$ and $\left.\varepsilon_{\mathrm{r}}<0\right)$, reduction of the film thickness leads to stiffening of the nanostructure.

5.2 Effect of External Loading. Consider the effect of external loading, which has always been neglected in the previously theoretical and numerical studies. Figure 7 shows the relationship between the normalized biaxial moduli of copper and silver metallic nanofilms and the film thickness under differently external loading strains. It is interesting that, for small external strain $\varepsilon$, the nanofilm stiffens with decreasing film thickness. For relatively large $\varepsilon$, the nanofilm becomes softer with a decreasing film thickness. It is easy to show that the interesting transition between stiffening and softening results from competition between the residual surface strain induced by surface relaxation and the excess surface strain due to external loading. For small external tensile loading, the residual compressive strain governs the elastic behavior of the copper or silver nanofilm. However, for large external tensile loading, the excess surface strain induced by tensile loading is the dominant factor and results in an increase in the interatomic 

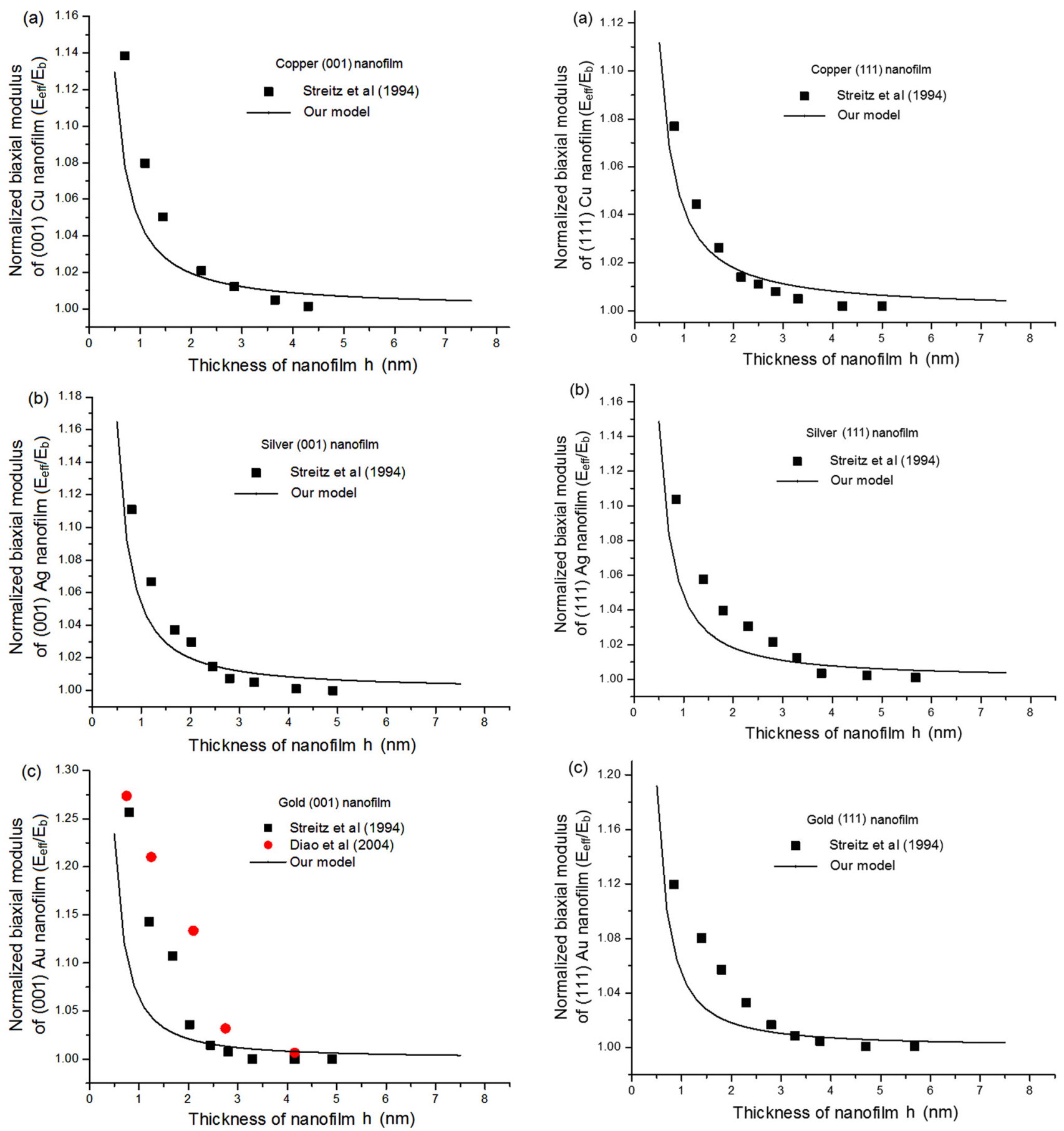

Fig. 5 Normalized biaxial moduli of different metallic nanofilms with $\{001\}$ free surface as a function of film thickness: (a) copper, (b) silver, and (c) gold

Fig. 6 Normalized biaxial moduli of different metallic nanofilms with $\{111\}$ free surface as a function of film thickness: (a) copper, (b) silver, and (c) gold

$$
E_{\text {eff }}=E_{b}+\frac{1}{a}\left(2 K^{s}+\Gamma_{11} \chi\right)
$$

[85]. All the results demonstrate that the present theory can predict not only the stiffening of a nanomaterial but also its softening.

\subsection{Comparison of Present Theory to Existing Theories}

5.3.1 Effective Young's Modulus. To compare the present theory to the existing theories, we focus on the effective Young's modulus of a nanomaterial.

In Dingreville et al. [36], the effective biaxial modulus of a metal nanofilm was expressed as

where $E_{b}$ is the bulk biaxial modulus, $K^{s}, \Gamma_{11}$, and $\chi$ are parameters related to the surface elastic constants and surface residual stress of a nanomaterial, and $a$ is the nanofilm thickness. To predict the effective biaxial modulus, the surface elastic constants that are used in surface elastic theory must first be determined by MD simulations.

In Chhapadia et al. [26], the effective Young's modulus of a nanowire was given as 

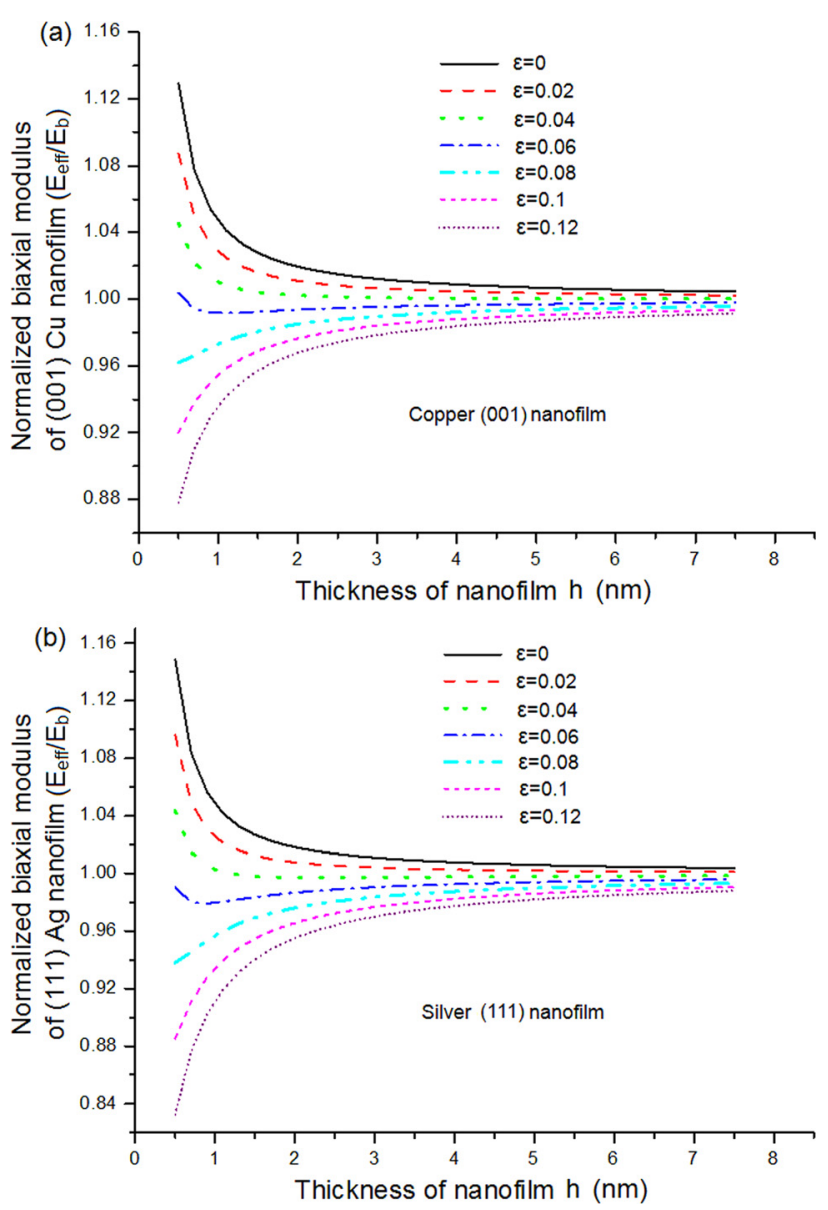

Fig. 7 Normalized biaxial moduli of different metallic nanofilms subjected to biaxial tension as a function of film thickness: (a) copper film with a $\{001\}$ free surface and (b) silver film with a $\{111\}$ free surface

$$
E_{\mathrm{eff}}=E_{b}\left(1+\frac{E_{s} I_{s}}{E_{b} I}+\frac{D_{s} I_{\mathrm{c}}}{E_{b} I}\right)
$$

where $E_{s}$ and $D_{s}$ are the surface elastic and bending moduli, respectively, $E_{b}$ is the Young's modulus of the corresponding bulk material, and $I$ and $I_{s}$ are the moment and perimeter moment of inertias of the nanowire's cross section, respectively. The quantity $I_{\mathrm{c}}$ is given by $I_{\mathrm{c}}=\int_{C_{w w}} n_{v}^{2} d C$, where $n_{v}$ is the vertical component of the unit normal vector perpendicular to the deflected nanowire and $C_{n w}$ denotes the perimeter of the nanowire. For theoretical predictions, MD simulation was used to obtain $E_{s}=-6.07 \mathrm{~N} / \mathrm{m}$ and $D_{s}=-6.77 \times 10^{-18} \mathrm{~N} \cdot \mathrm{m}$.

To predict the interface effect on the mechanical property of a nanocomposite, discontinuous conditions of interface stresses were introduced into the mesomechanics model in Duan et al. [40].

$$
\begin{aligned}
\mathbf{n} \cdot[\boldsymbol{\sigma}] \cdot \mathbf{n} & =-\boldsymbol{\sigma}^{s}:\left(\mathbf{n} \otimes \nabla_{s}\right) \\
(\mathbf{I}-\mathbf{n} \otimes \mathbf{n}) \cdot[\boldsymbol{\sigma}] \cdot \mathbf{n} & =-\boldsymbol{\sigma}^{s} \cdot \nabla_{s}
\end{aligned}
$$

where $[\boldsymbol{\sigma}]=\boldsymbol{\sigma}^{+}-\boldsymbol{\sigma}^{-}$denotes the stress difference across an interface, $\mathbf{n}$ is a unit vector in the normal direction of the interface, and $\nabla_{s}$ is an interface gradient operator. The stress tensor $\boldsymbol{\sigma}^{s}$ at the interface was assumed to satisfy a linearly elastic constitutive relation [20].

$$
\boldsymbol{\sigma}^{s}=\sigma_{0} \mathbf{I}+\mathbf{C}_{s}: \boldsymbol{\varepsilon}^{s}
$$

where $\sigma_{0}$ is the surface residual stress, $\boldsymbol{\varepsilon}^{s}$ is a surface strain tensor, and $\mathbf{C}_{s}$ is a fourth-order tensor of surface or interface elastic constants.

For a nanofilm under a biaxial tension, the biaxial modulus predicted by the present theory is

$$
E_{\text {eff }}=E_{b}\left(1-\frac{2 f_{1}}{E_{b} h}-\frac{2 f_{2} \varepsilon}{E_{b} h}\right)
$$

(a)
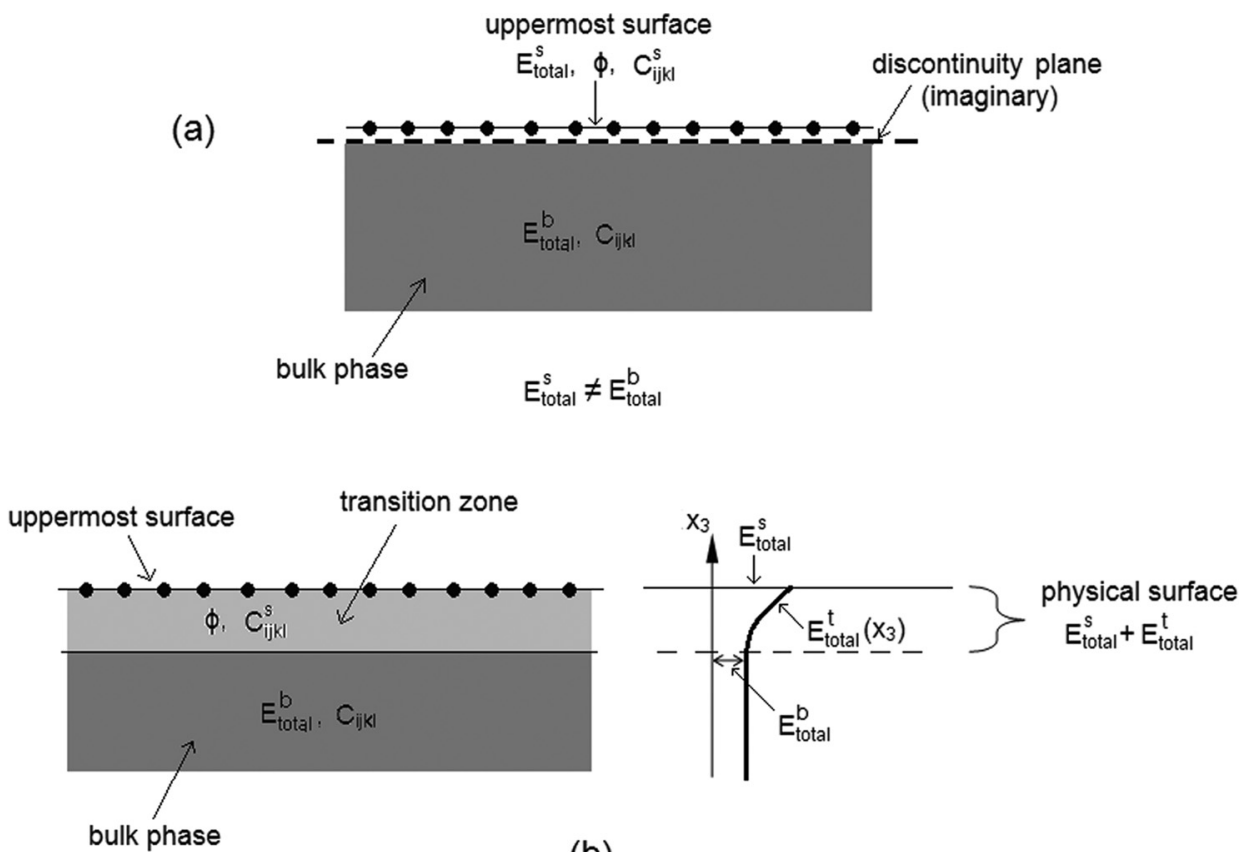

(b)

Fig. 8 Schematic of a nanosolid surface. (a) Zero-thickness surface defined in surface elastic theory. (b) Top surface layer and a transition zone (physical surface) in atomistic simulations. 
where the bulk surface-energy density $\phi_{0 \mathrm{~b}}$ and surface relaxation parameter $\lambda_{i}$ are involved, both of which are easy to determine via experiments and simple atomistic calculations.

5.3.2 Determination of Surface Parameters. According to Shenoy [49], the surface elastic tensor $C_{i j k l}^{s}$ can be formulated in terms of the Eulerian surface energy density $\phi$

$$
C_{i j k l}^{s}=\left.\left(-2 \phi \delta_{i k} \delta_{j l}+\delta_{i j} \frac{\partial \phi}{\partial \varepsilon_{k l}}+\frac{\partial^{2} \phi}{\partial \varepsilon_{i j} \partial \varepsilon_{k l}}\right)\right|_{\varepsilon=0}
$$

where $\delta_{i k}$ is the Kronecker delta symbol and the subscripts $i, j, k, l$ range from 1 to $2 ; \varepsilon$ is the strain tensor induced by an external loading with $\varepsilon_{i j}$ denoting its components. Based on Eq. (61), the surface elastic constants can be obtained by calculating the surface energy density $\phi$ as a function of strain $\varepsilon_{i j}$. However, the first and second derivatives of $\phi$ with respect to $\varepsilon_{i j}$ are rather cumbersome to achieve. Moreover, determinations of the 16 components of the surface elastic tensor $C_{i j k l}^{s}$ require high computational costs [49,54].

The surface parameters involved in our theory are the bulk surface-energy density and the surface-relaxation parameter, both of which can be obtained from experiments [86,87] or simple density functional theory (DFT) and MD simulations [54,88,89]. Moreover, the two involved quantities are scalars, which are much easier to determine than the fourth-rank tensor $C_{i j k l}^{s}$ in surface elastic theory.

5.3.3 Difference in Theoretical and Numerical Models. Both the existing surface elastic theories and the present one assume that the surface of a nanomaterial has a vanishing thickness $[20,21,36,37]$. It is usually regarded as a planar array of atoms attached to a nanosolid. A set of surface elastic constants are used to characterize its stiffness. A discontinuous interface exists artificially between the zero-thickness surface and the inside, as shown schematically in Fig. 8(a). The stiffness jumps from the inside to the surface at the interface.

In the atomic model, a transition zone consisting of several atomic layers should exist between the top atomic layer and the inside region. The elastic constants in the atomic model vary continuously through the transition zone from the top atomic layer to the inside layers, as shown schematically in Fig. 8(b).

By comparing the surface definitions in the theoretical models and the atomic simulation models, we find that the surface elastic constant in the previous theoretical models is really an effective parameter because it includes contributions from both the top atomic layer and from the transition zone in an atomic simulation model.

\section{Conclusions}

A new theory that considers surface effect of nanomaterials within the framework of continuum mechanics is established in this paper. The assumption of a linearly elastic constitutive relationship is not required any more. The surface-induced traction is found to be a function of surface-energy density instead of being a function of surface stress. With the relationship between the Eulerian description and the Lagrangian description of the surface-energy density and considering the influence of external loading on surface strain, we find that only two independent parameters, the surface-energy density of a bulk material and the surface relaxation parameter, are needed to characterize the surface effect in nanomaterials. Both parameters have clear physical meanings and are also very easy to determine by experiment or simple MD simulation. The theoretical prediction of the effective biaxial modulus of an fcc metallic nanofilm subjected to biaxial tension agrees very well with the existing MD result. An interesting finding is that the whole property of nanomaterials may exhibit nonlinearly elastic due to the nonlinear boundary conditions on surface though the inside of nanomaterials is linearly elastic. It can be inferred that the nonlinearly elastic property of nanomaterials may become stronger and stronger when the external loading increases or the characteristic size of nanomaterials decreases. Furthermore, it is found that the stiffening or softening behavior of a nanofilm results essentially from the competition between the residual surface strain induced by the surface relaxation and the excess surface strain caused by external loading. The present theory should be much convenient for predicting the mechanical behavior of nanomaterials, since the involved parameters are easy to ascertain. Further studies on surface and interface effects in many other nanosystems are forthcoming.

\section{Acknowledgment}

The work reported here was supported by the Natural Science Funds of China (NSFC) through Grants Nos. 11372317 and 11125211, the Nanoproject (2012CB937500), and the CAS/SAFEA International Partnership Program for Creative Research Teams. S.C. is much obligated to Professor Huajian Gao for helpful discussions.

\section{References}

[1] Ibach, H., 1997, "The Role of Surface Stress in Reconstruction, Epitaxial Growth and Stabilization of Mesoscopic Structures," Surf. Sci. Rep., 29(5-6), pp. $195-263$.

[2] He, L. H., Lim, C. W., and Wu, B. S., 2004, "A Continuum Model for Size-Dependent Deformation of Elastic Films of Nano-Scale Thickness," Int. J. Solids Struct., 41(3-4), pp. 847-857.

[3] Muller, P., and Saul, A., 2004, "Elastic Effects on Surface Physics," Surf. Sci. Rep., 54(5-8), pp. 157-258.

[4] Sander, D., 2003, "Surface Stress: Implications and Measurements," Curr. Opin. Solid State Mater. Sci., 7(1), pp. 51-57.

[5] Miller, R. E., and Shenoy, V. B., 2000, "Size-Dependent Elastic Properties of Nanosized Structural Elements," Nanotechnology 11(3), pp. 139-147.

[6] Liang, L. H., and Li, B. W., 2006, "Size-Dependent Thermal Conductivity of Nanoscale Semiconducting Systems," Phys. Rev. B, 73(15), p. 153303.

[7] He, Q. L., and Lilley, C. M., 2012, "Resonant Frequency Analysis of Timoshenko Nanowires With Surface Stress for Different Boundary Conditions," J. Appl. Phys., 112(7), p. 074322.

[8] Yan, Z., and Jiang, L. Y., 2012, "Vibration and Buckling Analysis of a Piezoelectric Nanoplate Considering Surface Effects and In-Plane Constraints," Proc. R. Soc. London A, 468(2147), pp. 3458-3475.

[9] Hall, B. D., Flueli, M., Monot, R., and Borel, J. P., 1991, "Multiply Twinned Structures in Unsupported Ultrafine Silver Particles Observed by Electron Diffraction,” Phys. Rev. B, 43(5), pp. 3906-3917.

[10] Kondo, Y., Ru, Q., and Takayanagi, K., 1997, "Thickness Induced Structural Phase Transition of Gold Nanofilm," Phys. Rev. Lett., 82(4), pp. 751-754.

[11] Mikkelsen, A., Skold, N., Ouattara, L., Borgstrom, M., Andersen, J. N., Samuelson, L., Seifert, W., and Lundgren, E., 2004, "Direct Imaging of the Atomic Structure Inside a Nanowire by Scanning Tunnelling Microscopy," Nat. Mater., 3(8), pp. 519-523.

[12] Hilner, E., Lundgren, E., and Mikkelsen, A., 2010, "Surface Structure and Morphology of InAs( 111$) \mathrm{B}$ With/Without Gold Nanoparticles Annealed Under Arsenic or Atomic Hydrogen Flux," Surf. Sci., 604(3-4), pp. 354-360.

[13] Cuenot, S., Fretigny, C., Champagne, S. D., and Nysten, B., 2004, "Surface Tension Effect on the Mechanical Properties of Nanomaterials Measured by Atomic Force Microscopy," Phys. Rev. B, 69(16), p. 165410.

[14] Chen, Y. X., Dorgan, B. L., Mcllroy, D. N., and Aston, D. E., 2006, "On the Importance of Boundary Conditions on Nanomechanical Bending Behavior and Elastic Modulus Determination of Silver Nanowires," J. Appl. Phys., 100(10), p. 104301.

[15] Jing, G. Y., Duan, H. L., Sun, X. M., Zhang, Z. S., Xu, J., Li, Y. D., Wang, J. X., and Yu, D. P., 2006, "Surface Effects on Elastic Properties of Silver Nanowires: Contact Atomic-Force Microscopy," Phys. Rev. B, 73(23), p. 235409.

[16] Gavan, K. B., Westra, H. J. R., van der Drift, E. W. J. M., Venstra, W. J., and van der Zant, H. S. J., 2009, "Surface Effects on Elastic Properties of Silver Nanowires: Contact Atomic-Force Microscopy," Appl. Phys. Lett., 94(23), p. 233108.

[17] Zhu, Y., Qin, Q. Q., Xu, F., Fan, F. R., Ding, Y., Zhang, T., Wiley, B. J., and Wang, Z. L., 2012, "Size Effects on Elasticity, Yielding, and Fracture of Silver Nanowires: In Situ Experiments," Phys. Rev. B, 85(4), p. 045443.

[18] Gibbs, J. W., 1928, Collected Works, Longmans Green, New York, p. 314

[19] Shuttleworth, R., 1949, "The Surface Tension of Solids," Proc. Phys. Soc. A, 63(5), pp. 444-457.

[20] Gurtin, M. E., and Murdoch, A. I., 1975, "A Continuum Theory of Elastic Material Surfaces,” Arch. Ration. Mech. Anal., 57(4), pp. 291-323. 
[21] Gurtin, M. E., and Murdoch, A. I., 1978, "Surface Stress in Solids," Int. J. Solids Struct., 14(6), pp. 431-440.

[22] Nix, W. D., and Gao, H., 1998, "An Atomic Interpretation of Interface Stress," Scr. Mater., 39(12), pp. 1653-1661.

[23] Haiss, W., 2001, "Surface Stress of Clean and Adsorbate-Covered Solids," Rep. Prog. Phys., 64(5), pp. 591-648.

[24] Murdoch, A. I., 2005, "Some Fundamental Aspects of Surface Modelling," J. Elasticity, 80(1-3), pp. 33-52.

[25] Steigmann, D. J., and Ogden, R. W., 1997, "Plane Deformations of Elastic Solids With Intrinsic Boundary Elasticity," Proc. R. Soc. London, Ser. A, 453(1959), pp. 853-877.

[26] Chhapadia, P., Mohammadi, P., and Sharma, P., 2011, "Curvature-Dependent Surface Energy and Implications for Nanostructures," J. Mech. Phys. Solids, 59(10), pp. 2103-2115.

[27] Wang, G. F., and Feng, X. Q., 2007, "Effects of Surface Elasticity and Residual Surface Tension on the Natural Frequency of Microbeams," Appl. Phys. Lett., 90(23), p. 231904

[28] Wang, G. F., and Feng, X. Q., 2009, "Surface Effects on Buckling of Nanowires Under Uniaxial Compression,” Appl. Phys. Lett., 94(14), p. 141913.

[29] He, J., and Lilley, C. M., 2008a, "Surface Effect on the Elastic Behavior of Static Bending Nanowires," Nano Lett., 8(7), pp. 1798-1802.

[30] He, J., and Lilley, C. M., 2008b, "Surface Stress Effect on Bending Resonance of Nanowires With Different Boundary Conditions," Appl. Phys. Lett., 93(26), p. 263108.

[31] Song, F., Huang, G. L., Park, H. S., and Liu, X. N., 2011, "A Continuum Model for the Mechanical Behavior of Nanowires Including Surface and Surface-Induced Initial Stresses," Int. J. Solids Struct., 48(14-15), pp. $2154-2163$.

[32] Lim, C. W., and He, L. H., 2004, "Size-Dependent Nonlinear Response of Thin Elastic Films With Nano-Scale Thickness," Int. J. Solids Struct., 46(11), pp. $1715-1726$.

[33] Lu, P., He, L. H., Lee, H. P., and Lu, C., 2006, "Thin Plate Theory Including Surface Effects," Int. J. Solids Struct., 43(16), pp. 4631-4647.

[34] Huang, D. W., 2008, "Size-Dependent Response of Ultra-Thin Films With Surface Effects," Int. J. Solids Struct., 45(2), pp. 568-579.

[35] Lachut, M. J., and Sader, J. E., 2007, "Effect of Surface Stress on the Stiffness of Cantilever Plates," Phys. Rev. Lett., 99(20), p. 206102.

[36] Dingreville, R., Qu, J. M., and Cherkaoui, M., 2005, "Surface Free Energy and Its Effect on the Elastic Behavior of Nano-Sized Particles, Wires and Films," J. Mech. Phys. Solids, 53(8), pp. 1827-1854.

[37] Dingreville, R., and Qu, J. M., 2008, "Interfacial Excess Energy, Excess Stress and Excess Strain in Elastic Solids: Planar Interfaces," J. Mech. Phys. Solids, 56(5), pp. 1944-1954.

[38] Huang, Z. P., and Wang, J., 2006, “A Theory of Hyperelasticity of Multi-Phase Media With Surface/Interface Energy Effect," Acta Mech., 182(3-4), pp. 195-210.

[39] Sharma, P., and Ganti, S., 2004, "Size-Dependent Eshelby's Tensor for Embed ded Nano-Inclusions Incorporating Surface/Interface Energies," ASME J. Appl. Mech., 71(5), pp. 663-671.

[40] Duan, H. L., Wang, J., Huang, Z. P., and Karihaloo, B. L., 2005, "SizeDependent Effective Elastic Constants of Solids Containing NanoInhomogeneities With Interface Stress," J. Mech. Phys. Solids, 53(7), pp. $1574-1596$.

[41] Huang, Z. P., and Sun, L., 2007, "Size-Dependent Effective Properties of a Heterogeneous Material With Interface Energy Effect: From Finite Deformation Theory to Infinitesimal Strain Analysis," Acta Mech., 190(1-4), pp. 151-163.

[42] Mogilevskaya, S. G., Crouch, S. L., and Stolarski, H. K., 2008, "Multiple Interacting Circular Nano-Inhomogeneities With Surface/Interface Effects," J. Mech. Phys. Solids, 56(6), pp. 2298-2327.

[43] Mogilevskaya, S. G., Crouch, S. L., Grotta, A. L., and Stolarski, H. K., 2010 "The Effects of Surface Elasticity and Surface Tension on the Transverse Overall Elastic Behavior of Unidirectional Nano-Composites," Compos. Sci. Technol., 70(3), pp. 427-434.

[44] Li, P. J., Wang, Q. Z., and Shi, S. F., 2011, "Differential Scheme for the Effective Elastic Properties of Nano-Particle Composites With Interface Effect," Comput. Mater. Sci., 50(11), pp. 3230-3237.

[45] Paliwal, B., and Cherkaoui, M., 2011, "Atomistic-Continuum Interphase Model for Effective Properties of Composite Materials Containing NanoInhomogeneities," Philos. Mag., 91(30), pp. 3905-3930.

[46] Paliwal, B., and Cherkaoui, M., 2012, "Estimation of Anisotropic Elastic Properties of Nanocomposites Using Atomistic-Continuum Interphase Model," Int J. Solids Struct., 49(18), pp. 2424-2438.

[47] Gao, X., Huang, Z. P., Qu, J. M., and Fang, D. N., 2014, "A CurvatureDependent Interfacial Energy-Based Interface Stress Theory and Its Applications to Nano-Structured Materials: (I) General Theory," J. Mech. Phys. Solids, 66, pp. 59-77.

[48] Wang, J. X., Huang, Z. P., Duan, H. L., Yu, S. W., Feng, X. Q., Wang, G. F., Zhang, W. X., and Wang, T. J., 2011, "Surface Stress Effect in Mechanics of Nanostructured Materials," Acta Mech. Solida. Sin., 24(1), pp. 52-82.

[49] Shenoy, V. B., 2005, "Atomistic Calculations of Elastic Properties of Metallic FCC Crystal Surfaces," Phys. Rev. B, 71(9), p. 094104.

[50] Streitz, F. H., Sieradzki, K., and Cammarata, R. C., 1990, "Elastic Properties of Thin FCC Films," Phy. Rev. B, 41(17), pp. 12285-12287.

[51] Streitz, F. H., Cammarata, R. C., and Sieradzki, K., 1994, "Surface-Stress Effects on Elastic Properties. I. Thin Metal Films," Phy. Rev. B., 49(15), pp. 10699-10706.
[52] Diao, J. K., Gall, K., and Dunn, M. L., 2004, “Atomistic Simulation of the Structure and Elastic Properties of Gold Nanowires," J. Mech. Phys. Solids, 52(9), pp. 1935-1962.

[53] Cao, G. X., and Chen, X., 2008, "Size Dependence and Orientation Dependence of Elastic Properties of $\mathrm{ZnO}$ Nanofilms," Int. J. Solids Struct., 45(6), pp. $1730-1753$.

[54] Mi, C. W., Jun, S., Kouris, D. A., and Kim, S. Y., 2008, "Atomistic Calculations of Interface Elastic Properties in Noncoherent Metallic Bilayers," Phys. Rev. B, 77(7), p. 075425.

[55] On, B. B., Altus, E., and Tadmor, E. B., 2010, "Surface Effects in Non-Uniform Nanobeams: Continuum Versus Atomistic Modeling," Int. J. Solids Struct., 47(9), pp. 1243-1252.

[56] Pahlevani, L., and Shodja, H. M., 2011, "Surface and Interface Effects on Torsion of Eccentrically Two-Phase FCC Circular Nanorods: Determination of the Surface/Interface Elastic Properties Via an Atomistic Approach," ASME J. Appl. Mech., 78(1), p. 011011.

[57] Mohammadi, P., and Sharma, P., 2012, "Atomistic Elucidation of the Effect of Surface Roughness on Curvature Dependent Surface Energy, Surface Stress, and Elasticity," Appl. Phys. Lett., 100(13), p. 133110.

[58] Olsson, P. A. T., and Park, H. S., 2012 "On the Importance of Surface Elastic Contributions to the Flexural Rigidity of Nanowires," J. Mech. Phys. Solids, 60(12), pp. 2064-2083

[59] Gao, W., Yu, S. W., and Huang, G. Y., 2006, "Finite Element Characterization of the Size-Dependent Mechanical Behaviour in Nanosystems," Nanotechnology, 17(4), pp. 1118-1122.

[60] Park, H. S., and Klein, P. A., 2008, "Surface Stress Effects on the Resonan Properties of Metal Nanowires: The Importance of Finite Deformation Kinematics and the Impact of the Residual Surface Stress," J. Mech. Phys. Solids, 56(11), pp. 3144-4166.

[61] Liu, C., Rajapakse, R. K. N. D., and Phani, A. S., 2011, "Finite Element Modeling of Beams With Surface Energy Effects," ASME J. Appl. Mech., 78(3), p. 031014

[62] Sun, C. Q., Tay, B. K., Zeng, X. T., Li, S., Chen, T. P., Zhou, J., Bai, H. L., and Jiang, E. Y., 2002, "Bond-Order-Bond-Length-Bond-Strength (Bond-OLS) Correlation Mechanism for the Shape-and-Size Dependence of a Nanosolid," J. Phys.: Condens. Matter, 14(34), pp. 7781-7795.

[63] Sun, C. Q., 2003, "Oxidation Electronics: Bond-Band-Barrier Correlation and Its Applications," Prog. Mater. Sci., 48(6), pp. 521-685.

[64] Ouyang, G., Tan, X., and Yang, G. W., 2006, "Thermodynamic Model of the Surface Energy of Nanocrystals," Phy. Rev. B, 74(19), p. 195408

[65] Ouyang, G., Li, X. L., Tan, X., and Yang, G. W., 2008, "Surface Energy of Nanowires," Nanotechnology, 19(4), p. 045709.

[66] Ouyang, G., Wang, C. X., and Yang, G. W., 2009, "Surface Energy of Nanostructural Materials With Negative Curvature and Related Size Effects," Chem. Rev., 109(9), pp. 4221-4247.

[67] Jiang, Q., and Lu, H. M., 2008, "Size Dependent Interface Energy and Its Applications," Surf. Sci. Rep., 63(10), pp. 427-464.

[68] Huang, Z. P., 2002, Fundamentals of Continuum Mechanics, Higher Education, Beijing, China.

[69] Gazis, D. C., Herman, R., and Wallis, R. F., 1960, "Surface Elastic Waves in Cubic Crystals," Phys. Rev., 119(2), pp. 533-544.

[70] Methfessel, M., Hennig, D., and Scheffler, M., 1992, "Trends of the Surface Relaxations, Surface Energies, and Work Functions of the 4d Transition Metals," Phys. Rev. B, 46(8), pp. 4816-4829.

[71] Lu, H. M., and Jiang, Q., 2004, "Size-Dependent Surface Energies of Nanocrystals," J. Phys. Chem. B, 108(18), pp. 5617-5619.

[72] Liu, D., Lian, J. S., and Jiang, Q., 2009, "Surface Energy and Electronic Structures of Ag Quasi-Crystal Clusters," J. Phys. Chem. C., 113(4), pp. $1168-1170$.

[73] Jiang, Q., Li, J. C., and Chi, B. Q., 2002, "Size-Dependent Cohesive Energy of Nanocrystals," Chem. Phys. Lett., 366(5-6), pp. 551-554.

[74] Tolman, R. C., 1949, "The Effect of Droplet Size on Surface Tension," J. Chem. Phys., 17(3), pp. 333-337.

[75] Jiang, Q., Liang, L. H., and Zhao, D. S., 2001, "Lattice Contraction and Surface Stress of FCC Nanocrystals," J. Phys. Chem. B, 105(27), pp. 6275-6277.

[76] Chen, X. L., Ma, H. S., Liang, L. H., and Wei, Y. G., 2009, “A Surface Energy Model and Application to Mechanical Behavior Analysis of Single Crystals at Sub-Micron Scale," Comput. Mater. Sci., 46(3), pp. 723-727.

[77] Zhang, W. X., Wang, T. J., and Chen, X., 2010, "Effect of Surface/Interface Stress on the Plastic Deformation of Nanoporous Materials and Nanocomposites,” Int. J. Plast., 26(7), pp. 957-975.

[78] Chen, T. Y., Chiu, M. S., and Weng, C. N., 2006, "Derivation of the Generalized Young-Laplace Equation of Curved Interfaces in Nanoscaled Solids," J. Appl. Phys., 100(7), p. 074308.

[79] Liu, J. L., Mei, Y., Xia, R., and Zhu, W. L., 2012, "Large Displacement of a Static Bending Nanowire With Surface Effects," Physica E., 44(10), pp. 2050-2055.

[80] Young, T., 1805, "An Essay on the Cohesion of Fluids," Phil. Trans. R. Soc. London, 95, pp. 65-87.

[81] Jiang, Q., Zhao, D. S., and Zhao, M., 2001, "Size-Dependent Interface Energy and Related Interface Stress," Acta Mater., 49(16), pp. 3143-3147.

[82] Liang, L. H., You, X. M., Ma, H. S., and Wei, Y. G., 2010, "Interface Energy and Its Influence on Interface Fracture Between Metal and Ceramic Thin Films in Nanoscale," J. Appl. Phys., 108(8), p. 084317.

[83] Liang, H. Y., Upmanyu, M., and Huang, H. C., 2005, "Size-Dependent Elasticity of Nanowires: Nonlinear Effects," Phys. Rev. B, 71(24), p. 241403. 
[84] Sheng, H. W., Kramer, M. J., Cadien, A., Fujita, T., and Chen, M. W., 2011, "Highly Optimized Embedded-Atom-Method Potentials for Fourteen FCC Metals," Phys. Rev. B, 83(13), p. 134118.

[85] Kittel, C., 1956, Introduction to Solid State Physics, Wiley, Inc. New York, p. 85.

[86] Tyson, W. R., and Miller, W. A., 1977, "Surface Free Energies of Solid Metals Estimation From Liquid Surface Tension Measurements," Surf. Sci., 62(1), pp. 267-276.
[87] Lamber, R., Wetjen, S., and Jaeger, N. I., 1995, "Size Dependence of the Lattice Parameter of Small Palladium Particles," Phys. Rev. B, 51(16), pp. 10968-10971.

[88] Vitos, L., Ruban, A. V., Skriver, H. L., and Kollar, J., 1998, "The Surface Energy of Metals," Surf. Sci., 411(1-2), pp. 186-202.

[89] Luo, W. H., Hu, W. Y., Su, K. L., and Liu, F. S., 2013, "The Calculation of Surface Free Energy Based on Embedded Atom Method for Solid Nickel," Appl. Surf. Sci., 265(15), pp. 375-378. 